\title{
H1-4 Gene
}

National Cancer Institute

\section{Source}

National Cancer Institute. H1-4 Gene. NCI Thesaurus. Code C115353.

This gene is involved in chromatin fiber formation. 